\title{
OPTIMIZACIÓN DE LA CADENA DE DISTRIBUCIÓN DEL CONGLOMERADO PYMES DEL SECTOR CÁRNICO DE BOGOTÁ, D.C.- GESTIÓN DISEÑO DE LA RED DE DISTRIBUCIÓN*
}

\author{
Chain optimization cluster distribution sector smes meat \\ Bogota, D.C. - design management distribution network
}

NATALIA CAMPOS VÁSQUEZ**, DELANY GARCÍA ÁLVAREZ***, CARLOS VIVAS SERRATO****

Recibido: 10 de Noviembre de 2015. Aceptado: 30 de Noviembre de 2015

DOI: http://dx.doi.org/10.21017/rimci.2016.v3.n5.a6

\section{Resumen}

Este artículo pretende evidenciar las técnicas y buenas prácticas que se pueden implementar en un proceso logístico dentro de la cadena de suministro, apoyados en las encuestas realizadas a diversas PYMES del sector cárnico bovino en Bogotá D.C. por los estudiantes del semillero de investigación «Optimización de la cadena de distribución del conglomerado pymes del sector cárnico en Bogotá» enfocados en la red de distribución, direccionado por el docente investigador Guillermo Enrique Montes Paniza de la Corporación Universitaria Republicana líder de la investigación; de tal manera que se identifiquen las fallas en los procesos del sector para así sugerir la implementación de una adecuada red de distribución, puesto que el propósito de la industria es brindar productos de calidad al cliente, reflejado en beneficios a la compañía con la optimización de procesos y disminución de costos.
\end{abstract}

Palabras clave: buenas prácticas, calidad, procesos, red de distribución, sector cárnico, técnicas.

\begin{abstract}
The intent of this article is to show techniques and best practices that can be implemented in a logistics process inside a supply chain, based on surveys completed in several small businesses of the beef industry in Bogota by the students of the study group "Improvement of the distribution by the association of small beef businesses of Bogota. This are focused on the supply chain, led by the investigation instructor Guillermo Enrique Montes Paniza of the Coporación Universitaria Republicana who is the author of the article; in order to identify the errors in the companies of the industry to suggest an improved distribution chain, since the aim of the industry to provide quality products for their client, which will provide benefits for the company while creating process improvement and reduced costs.
\end{abstract}

Keywords: best practices, quality, process, distribution network, meat sector, techniques.

* Resultado del trabajo de semillero en el marco del proyecto: Identificación y caracterización logística del conglomerado Pymes de cárnicos de bovino en Bogotá D.C. del grupo de investigación O.C.A liderado por el Ingeniero Guillermo Montes Paniza del programa de Ingeniería Industrial de la Corporación Universitaria Republicana.

* Estudiante Ingeniería Industrial, décimo semestre, Corporación Universitaria Republicana (Bogotá, Colombia). Correo electrónico: nanita9313@gmail.com

***Estudiante Ingeniería Industrial, décimo semestre, Corporación Universitaria Republicana (Bogotá, Colombia). Correo electrónico: delagarcia@hotmail.com

****Estudiante Ingeniería Industrial, décimo semestre, Corporación Universitaria Republicana (Bogotá, Colombia). Correo electrónico: calbertvi@hotmail.com 


\section{INTRODUCCIÓN}

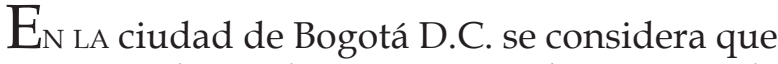
el consumo de productos cárnicos bovinos es de vital importancia para la canasta familiar, lo que conlleva a evidenciar la importancia de realizar un manejo apropiado del producto, para que llegue en óptimas condiciones al consumidor.

La logística como proceso fundamental interrelacional de una empresa, busca crear estrategias en la que cada uno de los procesos a través de una correcta planeación, implementación, control se vea beneficiado ya que al tener como herramienta fundamental la red de distribución se podrán parametrizar los procedimientos apropiados para la toma de las decisiones más influyentes y que se adecuen al modelo estratégico de la empresa.

Sin embargo, la red de distribución de la implementación de estrategias, es primordial para lograr exclusividad en los procesos con lo que puede competir en el mercado, por ello la importancia de conocer la conformación de cada uno de los factores en el diseño como la implementación de codificación de la información, conversión de datos en información, unidades de análisis, proceso de creación de perfiles, costos de las instalaciones y estimación de tarifas de transporte.

\section{II. ОвJеTIVo}

Evidenciar los procesos logísticos que ayudan a optimizar la cadena de distribución por medio de aplicaciones técnicas y buenas prácticas que sugirieran la implementación de una correcta planeación que disminuya costos y satisfaga al cliente con productos de calidad, apoyados en los resultados de encuestadas que se aplicaron al conglomerado PYMES del sector cárnico bovino de Bogotá D.C.

\section{Problema De investigación}

Actualmente Bogotá D.C. cuenta con algunas zonas específicas donde se localizan gran número de empresas del conglomerado PYMES del sector cárnico bovino, en ellas se evidencia la falta de estrategias enfocadas a la optimización de la cadena de distribución, esto derivado a que no hay una correcta recepción, almacenaje y disposición del producto hasta que es entregado al cliente, donde no necesariamente son los consumidores del producto terminado, pueden ser distribuidores mayorista, minoristas o incluso grandes superficies. Como lo expone Vatic Group2, en muchos casos el producto terminado de una cadena de suministro no es más que el inicio de otra, lo que expone la falta apropiación de técnicas y mejores prácticas, que disminuyan costos y ayude en la rentabilidad de la empresa [1].

Una buena planificación de la red de distribución es la vía más directa hacia la consecución de objetivos; pudiendo tratarse de metas relacionadas con el ahorro de costes o con aspectos relativos a la responsabilidad corporativa. En cualquier caso, es habitual que, incluso dentro del mismo sector, distintas empresas escojan un planteamiento de red de distribución diferente [2] como en caso de el Frigorífico San Martin, Frigorífico Guadalupe y el sector de la Calle 19 con Carrera 24, donde algunas de estas empresas buscan adaptar de otras, estrategias que afirman efectivas pero que no necesariamente les pueden servir, ya que no cuentan con herramientas y/o recursos suficientes para su implementación, en casos como los frigoríficos al ser una zona de alto consumo pueda que la agrupación geográfica incida en ventas, pero en algunos casos donde no se realiza un estudio adecuado del número y tamaño de almacenes, determinación de productos, cantidades a vender y costos de transporte, incurrirá en fallas que con el tiempo acarreará repercusiones no solo para la empresa sino para la satisfacción del cliente, ya que no cumplirá con los estándares de calidad esperados, con tiempo establecidos desde el momento de a relación comercial.

Por tanto para identificar las falencias que presentan las empresas pertenecientes al conglomerado PYMES del sector cárnico bobino, se expondrán los resultados de encuestas realizadas por el grupo del semillero «Optimización de la cadena de distribucion del conglomerado pymes del sector cárnico de Bogotá, D.C.» Subdivisión Gestión diseño de la red de distribución, donde se demuestra la inadecuada ejecución de la red de distribución por la falta conocimiento en cuanto a la red de distribución y sus componentes.

Rev. Ingeniería, Matemáticas y Ciencias de la Información Vol. 3 / Núm. 5 / enero-junio de 2016; pág. 57-70 
NATALIA CAMPOS VÁSQUEZ, DELANY GARCÍA ÁLVAREZ, CARLOS VIVAS SERRATO

Algunas de las preguntas tratadas en la investigación se enfocan en el proceso a seguir en la red de distribución, en donde el 30 \% de los encuestados argumentan que se debe a la ubicación, además de ser un sitio conocido, haber realizado estudios de tiempos y movimientos, estar cerca del lugar de sacrificio, contar con instalaciones y equipos de acuerdo a sus necesidades, adicionalmente, el $19 \%$ señala desconocer la información, otro 38 $\%$ argumenta que lo hace por tradición de los dueños de la compañía y el $13 \%$ lo para tener mayores ventas. Figura 1.

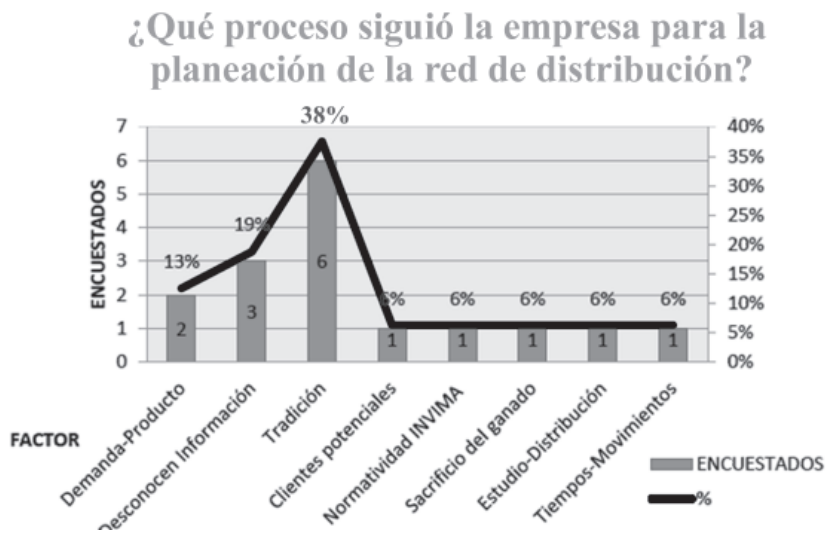

Fig. 1. Resultados - Proceso a seguir para la planeación de la red de distribución.

Sin embargo se alude el motivo de elección de la ubicación de la empresa en donde el 31\% (5 empresas) argumentaron que fue por la facilidad del sacrificio del ganado teniendo en cuenta que se encuentra dentro del frigorífico o cerca (San Martín - Guadalupe), el 25\% mencionan que se debe a los precios que obtienen en estos lugares, el 19\% su ubicación les genera un rentabilidad estable, el 13\% se debe al respaldo que tienen e obtener producto garantizados y el 6\% recalca que simplemente se debe por que es una sucursal de su punto de venta o porque llevan mucho en el mercado (tradición) y saben como es el manejo de lugar. Figura 2.

La fuente de información que se utilizo para realizar la red de distribución de la empresa en donde el 44\% (7 empresas) argumentaron que el factor principal fue la ubicación del frigorífico y la competencia perfecta, el 38\% de los encuestados afirman que se debe al beneficio economico que tienen al tener un transporte efectivo del lugar de sacrificio a sus negocios y de su empresa a sus

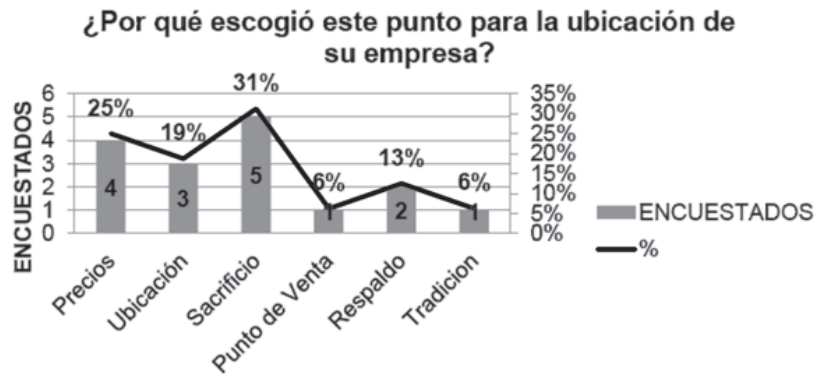

Fig. 2. Resultados - Punto de ubicación de la empresa.

clientes (si se requiriera) y el restante $19 \%$ afirma que es por tradición de la empresa que lleva muchos años en el mercado. Figura 3.

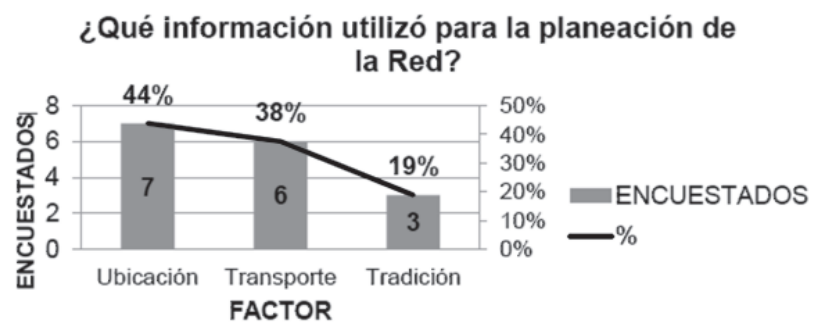

Fig. 3. Resultados - Información para la planeación de la Red.

También se cuestiona si las empresas del sector cuentan con codificación para sus productos y sus clientes, en donde más del $80 \%$ de los encuestados dicen tener codificados los clientes, de los cuales un 73\% afirman clasificarlos por C.C. ó NIT, mientras que un $7 \%$ expresó contar con un programa de fidelización el cual puede determinar sus necesidades a partir de las compras realizadas, en cambio el 20\% restante dijo no tener codificados sus clientes puesto que ya saben cómo identificarlos; en cuanto a los productos el $88 \%$ afirma tenerlos codificados mediante un software que utiliza la empresa y el $13 \%$ afirma que debido al volumen de ventas no es necesario contar con tanta tecnología, puesto que los empleados deben conocer muy bien los productos que están vendiendo. Figura 4.

Una de las herramientas más influyentes en la red de distribución es el transporte por lo que tambien se indaga el tipo de transporte que utilizan, a lo que el 75\% (12 empresas) manifestaron utilizar terrestres y lo hacen por medio de furgones (propios en sus mayoría), el 19\%, em- 


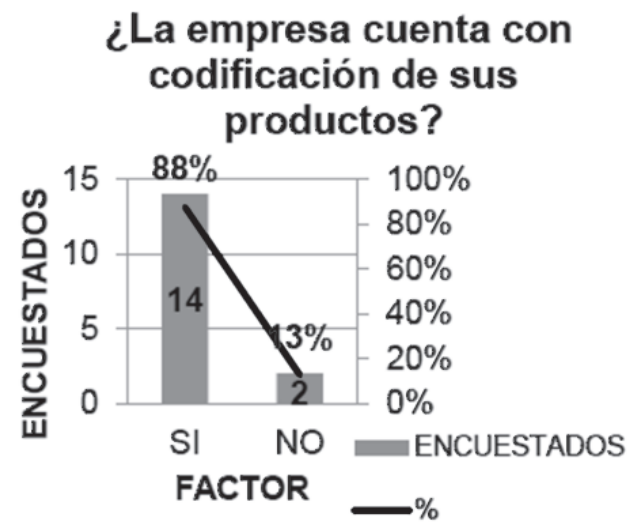

\section{¿La empresa cuenta con codificación de sus Clientes?}

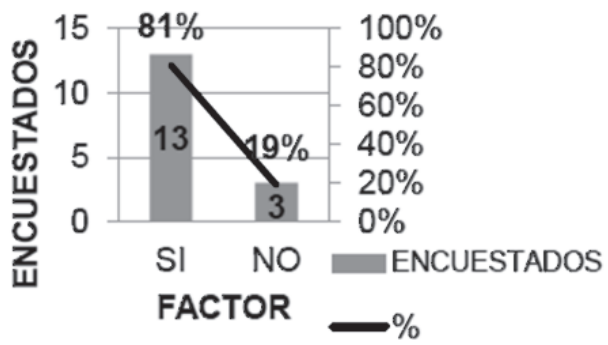

Fig. 4. Resultados - Codificación de los productos y clientes.

plear medios como motos cuanto no son grandes cantidades y a lugares no muy lejanos, claramente con neveras termicas, y el $6 \%$ afirmo utilizar transporte externo a ellos ya que no cuentan con uno propio. Figura 5.

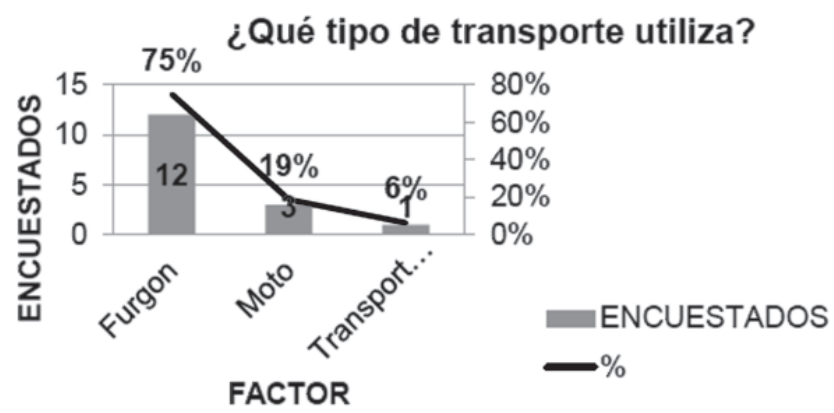

Fig. 5. Resultados - Tipo de transporte.

Y no menos importante saber el tipo de limitaciones que encontraron en el momento de realizar la planeación del diseño de la red, a lo que el 56\% afirmó no haber presentado ningún tipo de limitación, sin embargo el $44 \%$ reveló encontrar algunas a nivel financiero. Figura 6.

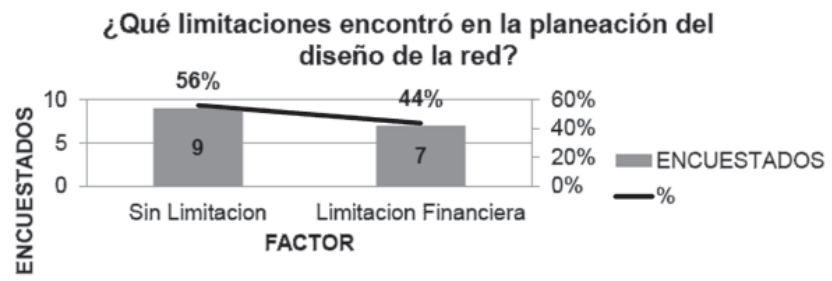

Fig. 6. Resultados - Limitaciones en la planeación del diseño de la red.
Adicional investigar la herramienta que emplean en el diseño de la red a los que un 56\% (9 empresas) manifestaron no haber tenido en cuenta ningún tipo de herramienta para su diseño, seguido de un $19 \%$ que dice no tener conocimiento o seguir una la red de otros almacén y finalmente un $6 \%$ afirma que desde la planeación del local ya se tenía claridad del tipo de herramientas a utilizar. Figura 7.

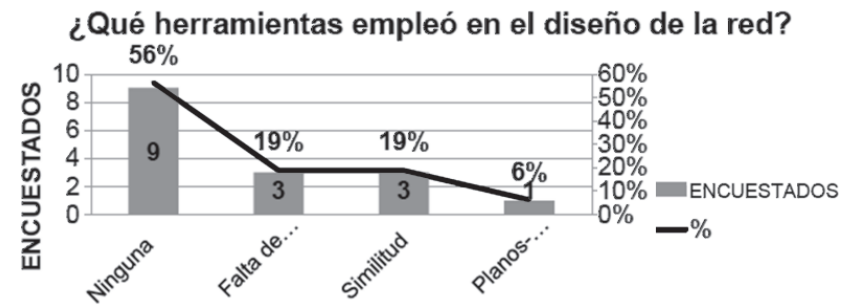

Fig. 7. Resultados - Herramientas empleadas en el diseño de la red.

Finalmente conocer acerca del número de almacenes o bodegas con que contaba la empresa para responder a la demanda de los consumidores del sector cárnico, a lo que 81\% (13 empresas) de los encuestados manifestaron contar con un solo almacén, argumentando que son empresas medianamente nuevas y que hasta ahora estaban incursionando en adquirir otro almacén o centro de distribución, sin embargo el 18\% de empresas restantes afirma tener uno o más locales en lugares de la ciudad donde saben que habrá buenas ventas. Figura 8.

Rev. Ingeniería, Matemáticas y Ciencias de la Información Vol. 3 / Núm. 5 / enero-junio de 2016; pág. 57-70 


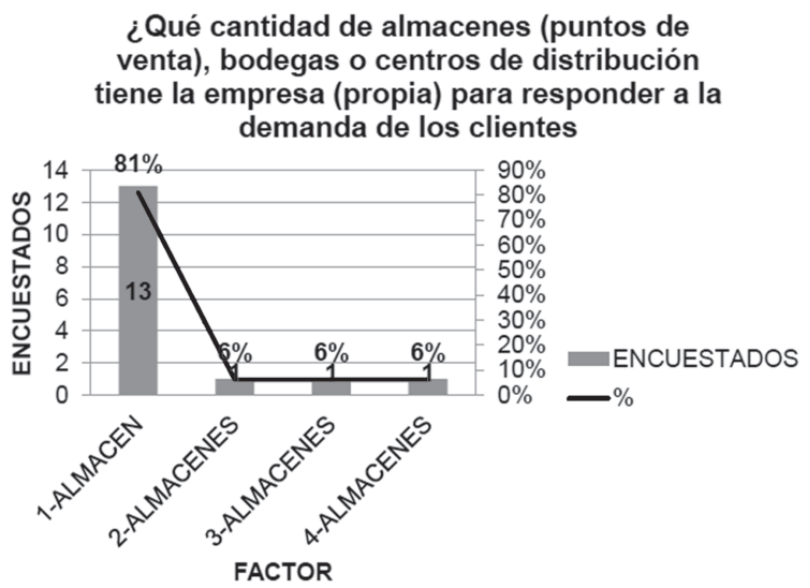

Fig. 8. Resultados - Cantidad de almacenes, bodegas o centros de distribución tiene la empresa.

\section{Gestión de la CADENA DE SUMINISTRO}

\section{A. Cadena de suministro}

Cada vez más, los mercados exigen costos bajos, alta calidad de productos y eficiencia en los tiempos de entrega. Una adecuada gestión dentro de la cadena de suministro debe ir perfilada hacía la entrega de productos de alta calidad, al precio justo y en el lugar correcto. Lograrlo implica que proveedores, fabricantes y vendedores, apliquen constantemente reingeniería en sus procesos funcionales y se implementen estrategias de colaboración a lo largo de la cadena de suministro [3].

Por lo anterior se apropia para esta investigación a la cadena de suministro como el conjunto de actividades que se desarrollan en la empresa con fin de que la materia prima sea transformada adecuadamente y así entregada al consumidor, ejerciendo el control en la ejecución de la optimización de los proceso, buscando una mejora económica, donde no se deje de lado el cumplimiento requerido para el manejo optimo y adecuado, que contribuye a la mejora de la calidad.

\section{B. Gestión de la cadena de suministro}

La cadena de suministro es aquella que «usa tecnología avanzada, gestión de la información e investigación de operaciones para planificar y controlar una complejidad creciente de factores para producir y entregar de mejor forma los productos y servicios para satisfacer el cliente» [2]. Por lo que caracteriza a la gestión de la cadena de suministro, como la integración de todas las planeaciones tecnológicas y de personal humano que se ejecutan el proceso para que el objetivo de la planeación sea alcanzado, verificando lo que se debe tener en un control logístico de actividades, conmutadas con el desarrollo de las operaciones y evitando que se produzcan errores que cambien los resultados esperados del cliente consumidor.

Sin embargo una cadena de suministro incluye un proceso de abastecimiento, fabricación y distribución, que opera conjuntamente con ventas, desarrollo de nuevos productos y marketing, siendo así un sistema complejo donde se busca que en la cooperación de las partes se logren establecer una relación compacta para su ejecución y más cuando la intervención de los procesos no solo es de los componentes internos sino de los cuales se unen todas las empresas que participan en la producción, manipulación, distribución, almacenamiento y comercialización del producto, contribuyendo en una relación que busca que todos los procesos cumplan estrictamente los requerimientos para que los resultados sean los productos de mejor calidad. Figura 9.

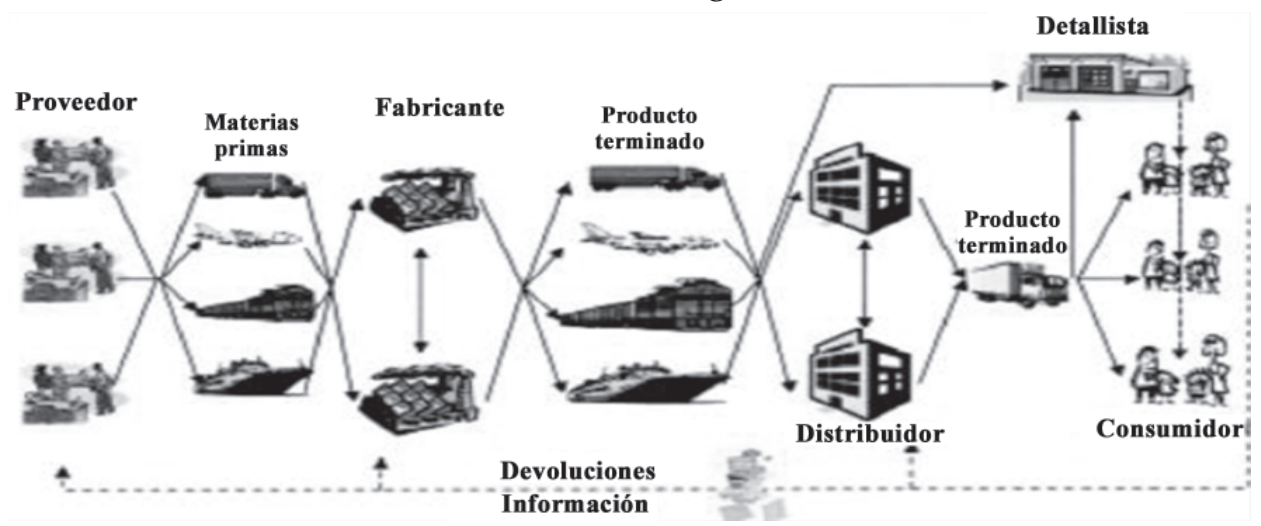

Fig. 9. Cadena de suministro básica. Fuente: Programa de Innovación Logística [4] 
Así también se puede complementar la estructura de la Fig. 9. Con el modelo de dirección de la cadena de suministro, en la que se puede identificar los principales factores que se deben de cumplir para que logre la satisfacción al cliente, esto con el propósito de llevar los bienes o servicios adecuadamente en el momento adecuado y con perfectas condiciones.

Por medio de la Fig. 9 se muestra la cadena de suministro básica y las relaciones que se generan por cada una de los integrantes que conforman el desarrollo para el cumplimiento de los requerimientos que exige el proceso de suministro en la importancia de cada uno de los influyentes ya que busca el propósito de llevar los bienes y servicios adecuadamente en el momento esperado y en perfectas condiciones. Figura 10.

Para Bowersox la gestión de la cadena de suministro «es una estrategia colaborativa para vincular operaciones de negocio inter-empresa para lograr una visión compartida de las oportunidades de negocio [2], por lo que al realizar un planteamiento estratégico que se ejecute en cada uno de los procesos de las empresas vinculas y con los que se pueda establecer una relación en la que se genere acuerdos con fines complementarios, las entidades relacionadas podrán adaptar estrategias que sean beneficiosas para cada una en la ejecución de los procesos.

Sin embargo se argumenta que la cadena de suministro de una entidad es clave en el entorno de la competitividad, por lo que si se establece, puede traer a la empresa ventajas que la acerquen a la efectividad, y es que cuando se identifica que dentro de la gestión de la cadena de suministro se debe de tener una dimensión estratégica con la que se puedan establecer componentes, se logrará una verdadera competitividad en cada uno de los procesos para lograr establecer puntos que lleguen a posesionar el producto, ya que establecida dicha estrategia a largo plazo, se identificará la dimensión tatica en la cual se deben de tomar decisiones que se visualizaran durante el proceso como consecuencia al cambio de actividades por realizar, como una innovación que impacte y pueda levantar procesos caídos que en ocasiones promuevan un producto nuevo, que igualmente traería a implementar la dimensión operativa, que evidencia las tareas operacionales de almacén, la producción o distribución, ya que se verá alterada, y en ese momento es donde la planeación de la dimensión estratégica se tangibiliza y se pone a prueba la decisión táctica, ya que actualmente son muy pocos los procesos que utilizan estratégicas operativas. Figura 11.

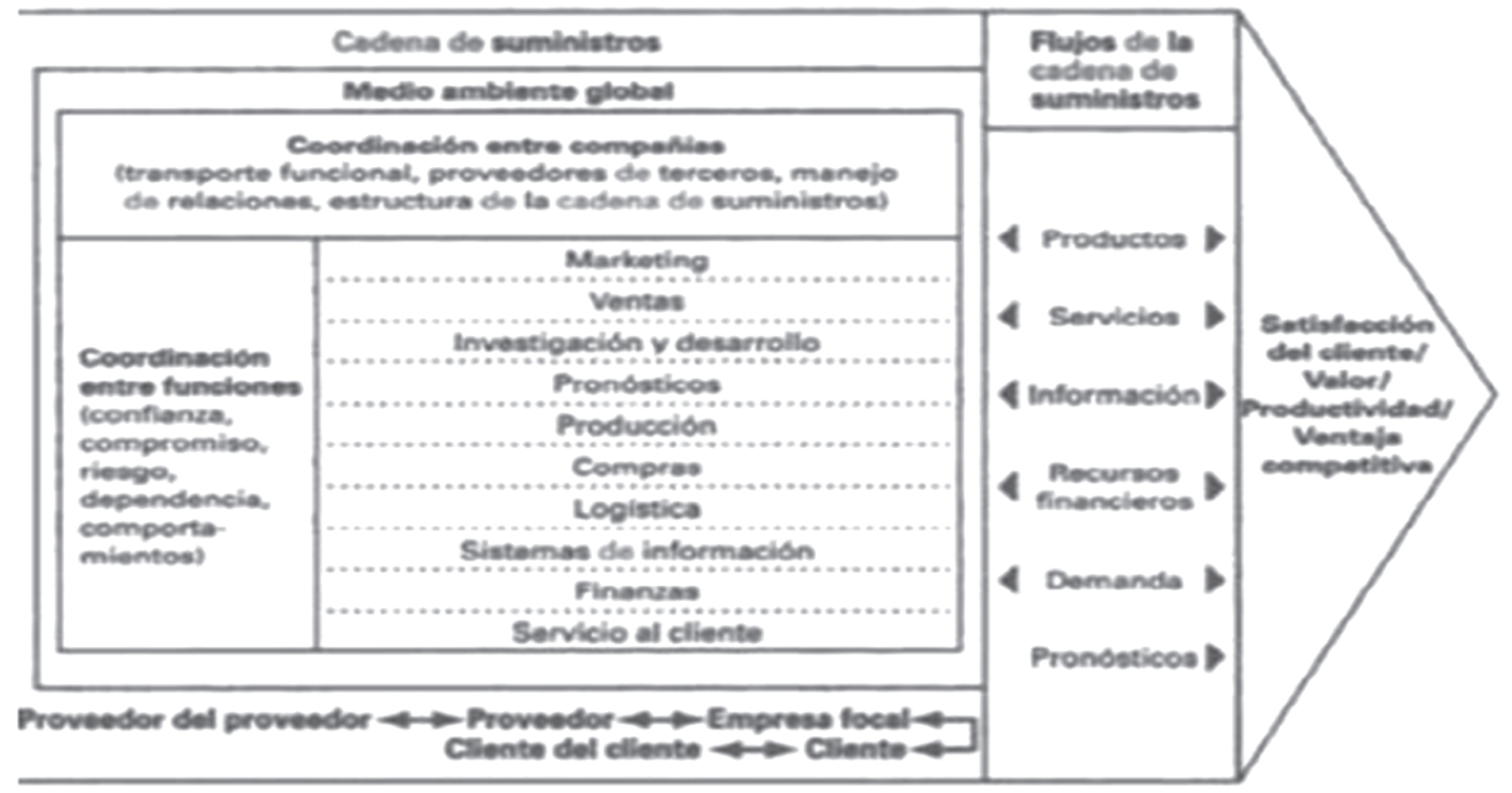

Fig. 10. Modelo de dirección de la cadena de suministro. Fuente: Logística administración de la cadena de suministro[5] 
NATALIA CAMPOS VÁSQUEZ, DELANY GARCÍA ÁLVAREZ, CARLOS VIVAS SERRATO

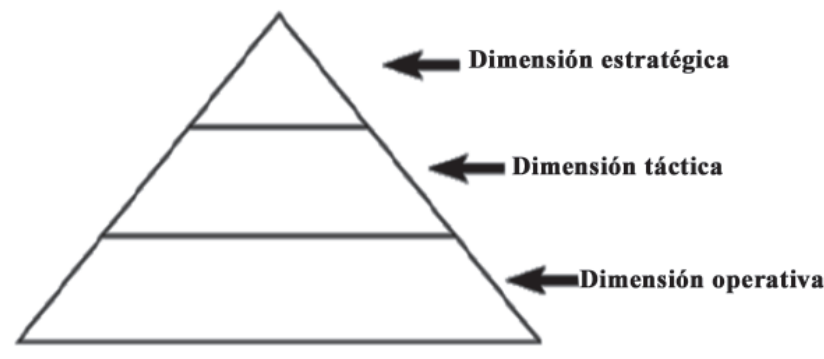

Fig. 11. Dimensiones de Supply Chain Management.

Fuente: Supply Chain Management segunda edición[2]

\section{Logística}

Toda empresa de cualquier sector (primario, secundario o terciario), está constituida por gran número de elementos como personal, materias primas, materiales, datos y recursos económicos, los cuales no se consideran elementos estáticos sino que fluyen bajo una estructura y funciones, por lo que se puede determinar que todos se mueven dentro de la organización, donde existe hay un flujo de personal, un flujo de material, un flujo económico del cual se deben derivar áreas para manejar la logística de todos estos elementos del sistema con el fin a alcanzar la misión de la empresa y poder incurrir a proponer la implementación del departamento de logística para que se puedan establecer tiempos y movimientos adecuados para ejercer las actividades que requiere la empresa, por lo que se define a la logística como la disciplina que debe implementar de manera oportuna el planear, gestionar, controlar y mejorar continuamente en cada uno de los procesos que se desarrollen en la empresa.

El factor localización espacial es uno de los elementos que condicionan la competitividad de una nación, por ende, este factor se ha incorporado al análisis a través de los costos de transporte que afectan los costos de producción de la cadena y, a su vez, el precio final del producto [6]; principalmente de este factor se debe desencadenar los componentes espaciales de distancia que separa al productor del consumidor final y de la infraestructura por la que se debe someter el transporte de los alimentos, estos factores refieren importancia ya que determinan el tipo de transporte que debe de utilizar, debido recorrido para la entrega, teniendo en cuenta que de ello se derivan los valores agregados de precio final al consumidor.

\section{Logística y la cadena de suministro}

La logística en la cadena de suministro se define como la parte encargada de planificar, implementar y controlar de forma eficiente y efectiva en el punto de almacenaje y el flujo directo e indirecto de los bienes y servicios dentro de la distribución directa e indirecta del punto de origen de los procesos para la ejecución de un proyecto [7].

Por lo que se puede afirmar que la logística busca conseguir que los productos y servicios se encuentren convenientemente en el lugar preciso, en el momento oportuno y en las condiciones, ya que las actividades logísticas de la empresa se deben centrar en 3 áreas básicas:

1) Proceso de aprovisionamiento, almacenamiento del material y los punto de la planta de proceso.

2) Proceso de producción, realización de las operaciones productivas en la planta.

3) Proceso de distribución, distribución estratégica del punto de almacenamiento del producto terminado al consumidor.

Por consiguiente la logística busca establecer un valor en tiempo y lugar, con fin de que en el momento que el consumidor adquiera el bien o servicio, encuentre el producto en el tiempo requerido y lugar indicado, lo que originará un beneficio tanto proveedores como accionistas de la empresa.

\section{E. Gestión estratégica}

Según James B. Quinn la gestión estratégica define "para casi todos a quienes se les pregunte, la estrategia es un plan, una especie de curso de acción conscientemente determinado, una guía (o serie de guías) para abordar una situación específica» [8].

\section{F. Estrategia de la logística y de la cadena de suministro}

«La selección de una efectiva estrategia de logística y de la cadena de suministro requiere algo del mismo proceso creativo necesario para desarrollar una adecuada estrategia corporativa. Los 
enfoques innovadores en la estrategia logística y de la cadena de suministro pueden representar una ventaja competitiva» [9].

Gran cantidad de empresas y sobre todo las grandes superficies emplean la estrategia de logística y de la cadena de suministros como un elemento esencial en su manejo estratégico para lograr establecer competencia en el mercado.

Por lo cual a muchos retos se debe someter a las empresas hoy en día, ya que no basta con hacer las cosas bien, si no se debe buscar hacerlas excelentes en todos los procesos, porque la creciente competitividad y globalización del mercado son los principales factores que someten a que una empresa a que cada día se vuelva más efectiva y estratégica en todos los procesos, visualizando un panorama donde se vea que el producir y comercializar no termina en la venta como tal, sino que es satisfactorio cuando el cliente final acepta y ha pagado por un producto que considera de buena calidad.

\section{G. Cadena de distribución}

Conformada por la relación de los flujos que transforman los bienes desde la etapa de la materia prima hasta el consumidor final, esta igualmente constituida por el flujo de información de las cuales se relacionan y se determinan las decisiones adecuadas para el desarrollo de los movimientos adecuados y oportunos.

En una cadena de distribución física se pueden identificar las siguientes actividades: ( Figura 12).

1) Aprovisionamiento de insumos

2) Producción y/o procesamiento

3) Inspección del bien
Cadena 1

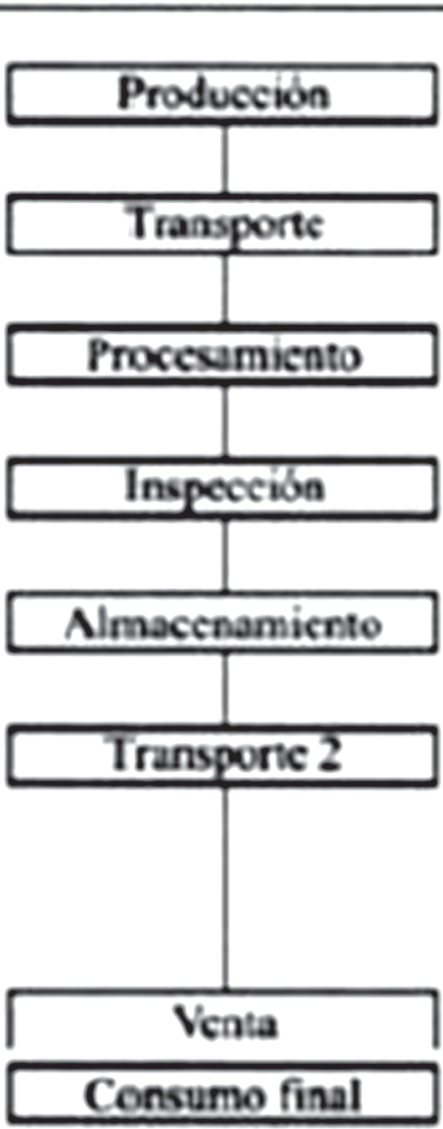

Cadena 2

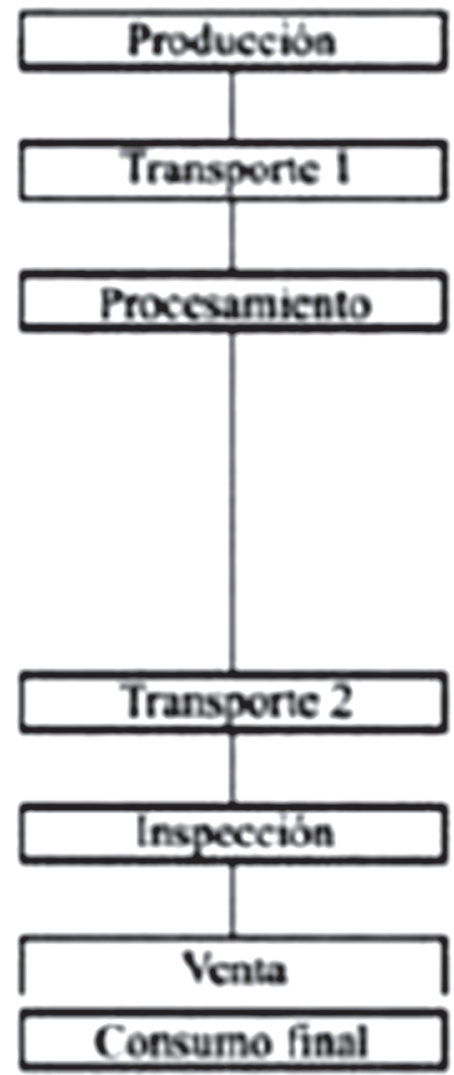

Cadena 3

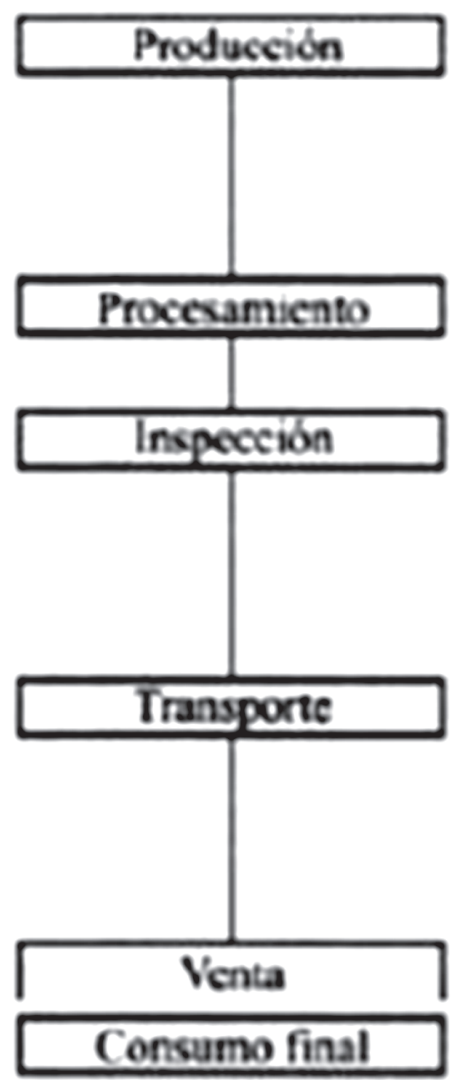

Fig. 12. Cadena de distribución física que se diferencia por las actividades. Fuente: Logística de distribución física a minoristas [10] 

NATALIA CAMPOS VÁSQUEZ, DELANY GARCÍA ÁLVAREZ, CARLOS VIVAS SERRATO

4) Transporte, almacenamiento y otras operaciones logísticas

5) Venta

6) Consumo fina (cliente consumidor)

Teniendo en cuenta que para la construcción de cada uno de las actividades se debe tener cada especificación acerca de las condiciones que determinan una óptima cadena de distribución, no se deben dejar de lado los elementos que la conforman como el «diseño de la red de distribución, almacén, manejo de inventarios y portafolio de productos, gestión del transporte, servicio al cliente, indicadores de gestión»[11].

\section{H. Diseño de la red de distribución}

En el diseño de una Red de Distribución se determina el número, ubicación, y capacidad de cada una de los elementos de la red de distribución (punto de venta, bodega, almacén, centro de distribución) de manera tal que sean mínimos los costos globales de fabricación, almacenamiento y transporte [1].

Agregando al diseño de la red de distribución se puede tomar referencia las buenas prácticas para aplicar en los factores de fabricación, almacenamiento y transporte para disminuir los costos siempre y cuando se cumplan con los requerimientos de calidad con el que se debe manejar la materia prima.

Para lograr una construcción adecuada de la planeación de la red, se toma como referencias las adecuaciones principales que se deben implementar según, Ballou las cuales identifica como la información de los elementos de bases de datos requeridos [5]:

1) Codificación de la información: se adecua para facilitar la información ya que se propone codificar los productos y los clientes, esto con el fin de que se pueda identificar los niveles de movimiento de los productos y de la frecuencia de los clientes, esto lleva a una decisión adecuada de transporte y de los inventarios.

2) Conversión de datos en información: denotar la agrupación que se requiere para que se encuentren acomodados y apoyen a la planeación de la red.

3) Unidades de análisis: decidir las dimensiones que serán utilizadas en el análisis: peso (lb o kg), medida monetaria (\$), contero físico (cajas, unidades).

4) Agrupación de productos: determinar según el manejo como se deben agrupar los productos ya sea por las características o por determinación reglamentaria del manejo de cada producto.

5) El proceso de crear perfiles [12]: se realiza para identificar rápidamente la causa de los problemas en el flujo de información y materiales, para indicar las principales oportunidades para hacer mejoras en los procesos y para construir una base sobre la cual se pueda tomar decisiones.

6) Estimación de la tarifa de transporte: identificación de las dimensiones de los productos, la distancia del punto de origen y el receptor, carga, almacenamiento y el tipo de transporte (propio o contratado).

7) Costos de las instalaciones: relacionados con una instalación: costo fijos, de almacenamiento, de manejo al igual que verificar como se afecta directamente los inventarios por el número, la cantidad y el tamaño del almacén.

\section{Almacén}

La Asociación Logística Europea considera que es un edificio especialmente proyectado para recibir, guardar, manipular, reacondicionar y expedir los productos que vende una organización [13].

En logística se ha buscado la manera de cómo lograr la optimización de la ubicación del almacén en función al costo del transporte, y se ha implementado modelos matemáticos con el fin de identificar una solución óptima y razonable al problema, teniendo en cuenta la entrada y salida.

Por lo que el modelo matemático adecuado para toma la decisión la cual se denomina como Centro de Gravedad, ya que logra identificar los principales factores como la demanda y localización adecuada donde se puedan optimizar los recorridos de transporte. Figura 13. 


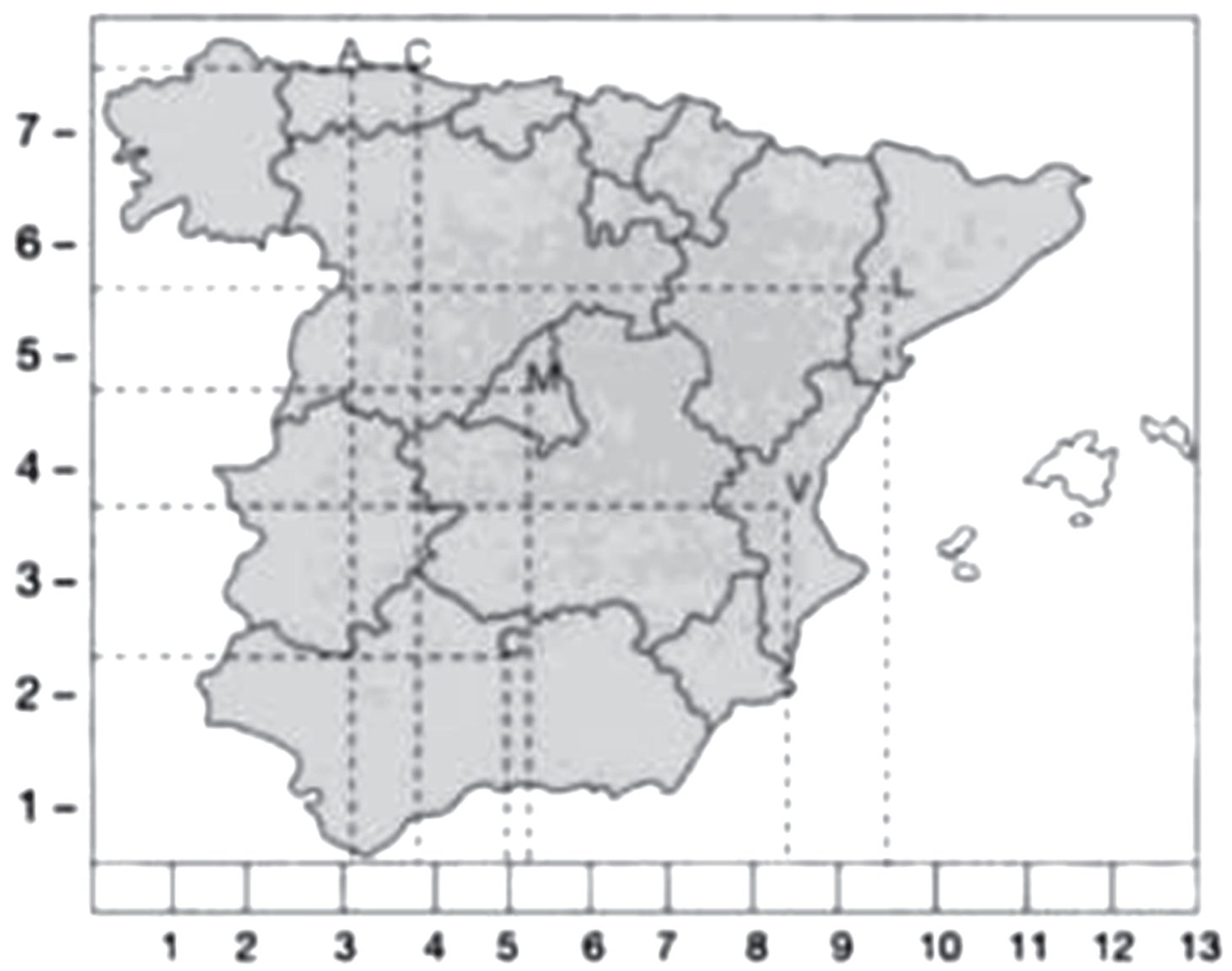

Fig. 13. Planteamiento de los puntos de conformación para la determinación del centro de gravedad.Fuente. Logística integral[14]

Incluyendo dentro de la ponderación los parámetros relativos del costo del transporte, este método es especialmente utilizado para tratar de definir la mejor posición del almacén central que es el encargado de recibir productos de diferentes proveedores y que así mismo son quienes abastecen la red de almacenes o centros de distribución.

La ubicación de instalaciones usualmente está determinada por un factor crítico [5]: la ubicación de la planta de producción y almacén está dominada por factores económicos, mientras que la ubicación de puntos de venta está gobernada por los ingresos que genera la ubicación, con los costos del sitio restados de los ingresos para determinar la rentabilidad.

En ese sentido Thünen determinó que la utilidad que se podría pagar por la ubicación de una tienda en una ciudad es la diferencia entre el precio de la tienda dentro de la ciudad y el costo de transportar los bienes a dicha tienda y que dichas tiendas se ubicarían lo más cercano al centro de la ciudad y a lo largo de las vías de transportación [15]. Por lo que utiliza la ecuación:

$$
R=Y(P-C p)-(C t x d)
$$

Donde:

$$
\begin{aligned}
& \mathbf{R}=\text { renta por unidad de tierra } \\
& \mathbf{P}=\text { precio de mercado del bien }(\$ / \text { ton }) \\
& \mathbf{Y}=\text { producto por unidad de tierra } \\
& \mathbf{C P}=\text { costo de producción del bien }(\$ / \text { ton }) \\
& \mathbf{C t}=\text { costo de transporte por unidad } \\
& \mathbf{d}=\text { de distancia }(\$ / \text { ton } / \text { miles })
\end{aligned}
$$

\section{MEJORES PRÁCTICAS}

La red de distribución puede llegar a ser la clave para adquirir ventajas competitivas y éxito en el mercado, minimizando costos operacio- 
NATALIA CAMPOS VÁSQUEZ, DELANY GARCÍA ÁLVAREZ, CARLOS VIVAS SERRATO

nales, carga impositiva y maximizar el servicio al cliente.

Pero ¿Cómo diseñar una red de distribución para maximizar el servicio al cliente, la flexibilidad y minimizar los costos operacionales?. Esto se puede determinar a través de un alineamiento con fundamentos básicos que se requieran los siguientes pasos donde se logre alcanzar los objetivos y eviten riesgos en la ejecución de la red:

1) Identificando el alcance y las restricciones de la red actual

Los alcances en volúmenes de ventas, bases de clientes, requerimientos.

2) Determinando metas

Identificando las problemáticas más relevantes, se debe enfocar en la mejor y más completa, así se podrá impulsar decisio- nes acerca de donde se debe concentrar las estrategias.

3) Recolectando datos acerca de la situación de la red actual

4) Limitando y verificando los datos recolectados.

5) Construyendo modelos de base (históricos)

6) Fundamentando un modelo de potenciales escenarios estratégicos de la red

Identifica si la red que se tiene propuesta está actuando de la mejor manera o si por lo contrario se pueda prestar para cambios específicos que mejoren los costos operacionales.

7) Preparando los Cambios Recomendados en la Red. Figura 14.

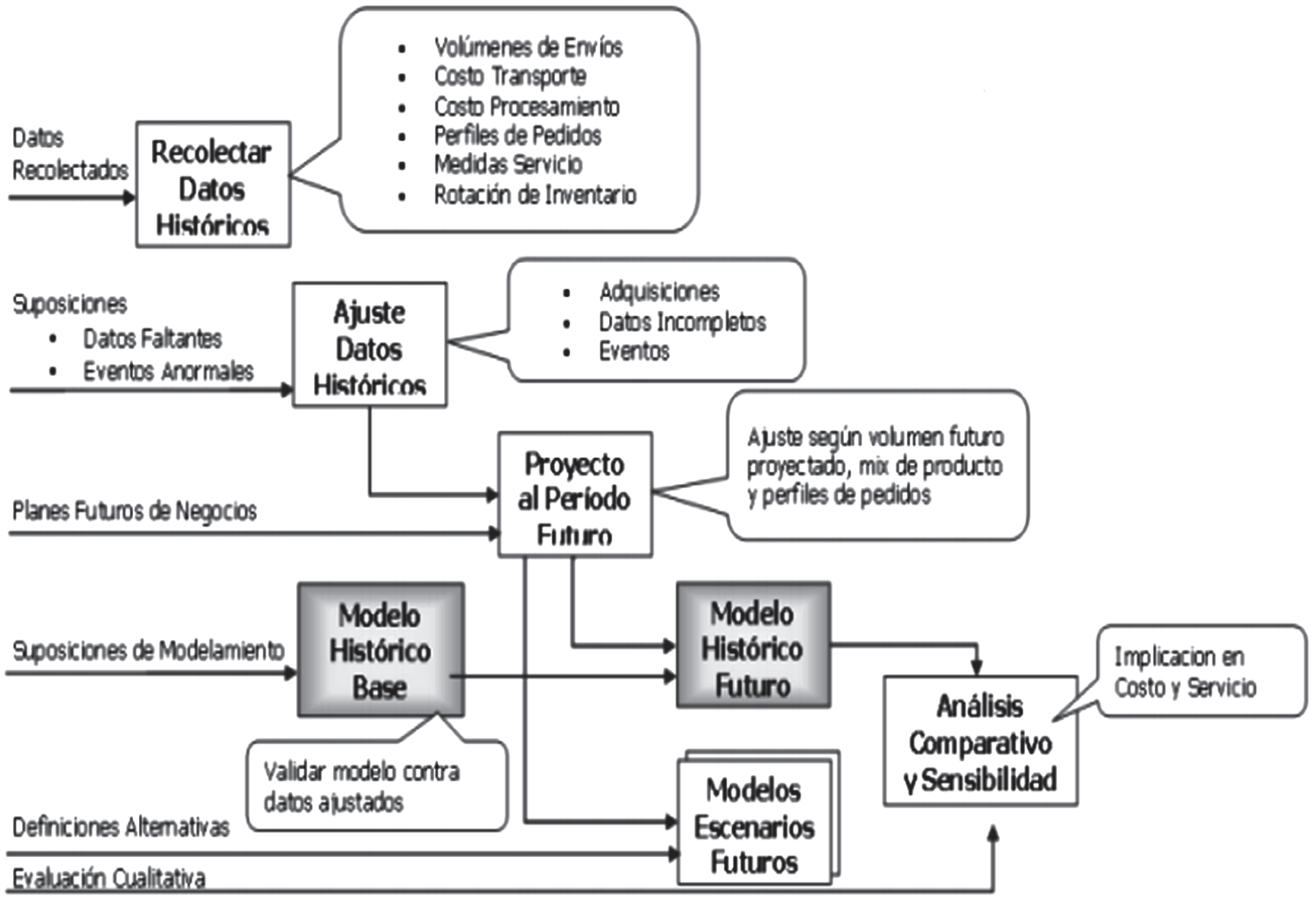

Fig. 14. Modelo de optimización de redes. Fuente: Fortna [16]. 


\section{MODELO DE OPTIMIZACIÓN DE REDES}

\section{A. Mi Pymes en Colombia}

Según la ley 590 de julio 10 de 2.000 para promover el desarrollo de las Micro, Pequeña y Mediana Empresa [17], las PYMES se clasifican de la siguiente manera:

\section{1) Microempresa}

Con personal no superior a 10 trabajadores, activos totales inferiores a 501 salarios mínimos mensuales legales vigentes; la ley 509 la modificó con planta de personal no superior a 10 trabajadores o activos totales excluida la vivienda por valor inferior a 500 salarios mínimos legales vigentes.

2) Pequeña empresa

Con personal entre 11 y 50 trabajadores; activos totales mayores a 501 y menores a 1.001 salarios mínimos mensuales legales vigentes; la ley 509 la modificó con planta de personal entre 11 y 50 trabajadores o activos totales entre 501 y menos de 5.000 salarios mínimos legales vigentes.

3) Mediana empresa

Con planta de personal entre 51 y 200 trabajadores; activos totales entre 5.001 y 15.000 salarios mínimos legales vigentes; posteriormente la ley 905 de 2.004 la modificó con planta de personal entre 51 y 200 trabajadores, o activos totales hasta 30.000 salarios mínimos legales vigentes.

\section{Sector cárnico en Colombia}

La estructura productiva del Sector Cárnico en Colombia inicia con la cría y engorde de ganado vacuno, ganado porcino, aves de corral y especies menores (ovino, caprino, conejos); continúa con el transporte, sacrificio, corte, congelación y comercialización de estos productos para la colocación de carnes en el mercado final, donde a la vez se generan subproductos como grasas, sebos y sangre, y termina con la elaboración de carnes embutidas, arregladas y frías [18].

\section{A. Importancia económica del sector cárnico en Colombia}

«Según estudios realizados por el DANE (Encuesta Anual Manufacturera) las industrias de alimentos, bebidas y tabaco, contribuyen al 33\% del total de la producción industrial del país, esta industria constituida por el desarrollo económico de diferentes productos: lácteo, bebidas alcohólicas, aceites, grasas, alimentos para animales, bebidas no alcohólicas, azucares y, carnes y derivados, estos últimos motivo de estudio del artículo que representa un $12 \%$ de la producción de los sub sectores, lo que contribuye de manera significativa al fortalecimiento de la industria del país, y acrecienta su importancia, por los altos índices de desarrollo, pues la carne colombiana es reconocida por su calidad y procesos de innovación aplicados en los últimos años» [19].

\section{B. Consumo de carne anual de carne en Colombia}

Según la Ley 1712 de Transparencia y del Derecho de Acceso a la Información Pública Nacional, se determina la información del consumo de carnes en Colombia durante los periodos graficados [20]. Figura 15:

\section{CONCLUSIONES}

La red de distribución es indispensable en el funcionamiento de una empresa, ya que al ejecutarse de manera precisa y concisa, junto con todos los suministros y materiales necesarios, se puede cumplir con los requerimientos de los procesos operativos que hacen parte de cada punto de la red.

Las herramientas de la red de distribución tienen como objeto lograr minimizar los costos, considerando factores como tiempo y nivel de servicio que se brinda al consumidor.

Al emplear de manera oportuna la red de distribución los favorecidos serán los clientes al obtener productos de calidad, en tiempos esperados en las condiciones pactadas.

Es importante identificar un modelo matemático adecuado que se pueda utilizar para ubicar los centros de distribución o puntos de venta de la 


\section{Consumo aparente per capita anual}

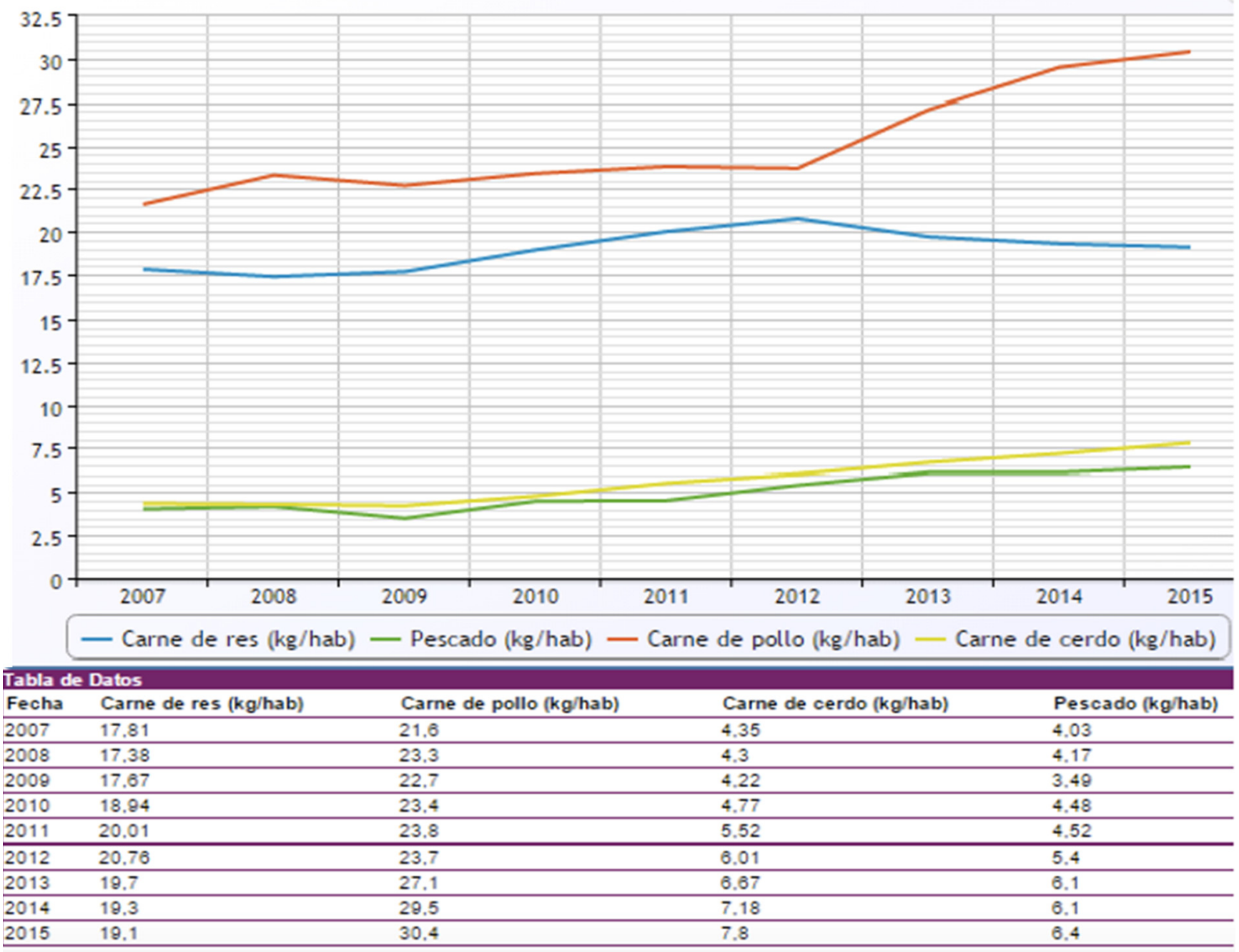

Fig. 15. Consumo aparente per cápita anual. Fuente: Fedegan [20]

empresa de acuerdo a las necesidades que la misma tenga.

Las buenas practicas se pueden implementar en la empresa de acuerdo a la necesidades que se tengan, ya que con estas se podrán afrontar las necesidades de manera oportuna y se generaran estrategias con las que se podrán tomar decisiones que optimicen la logística en la empresa.

Los resultados de esta investigación reflejan la falencia existente en la aplicación de una adecuada red de distribución, por lo que se propone la implementación de buenas prácticas en el cual se busca que el manejo y distribución de los productos cárnicos bovinos en Bogotá D.C. mejore, para obtener productos finales de alta calidad, beneficiando los procesos del conglomerado PYMES bobino.

\section{Agradecimiento}

A la Corporación Universitaria Republicana por abrir espacios de investigación donde se fomente realización de actividades formativas en el que la comunidad académica pueda interactuar con otras áreas, al docente investigador Guillermo Enrique Montes Paniza autor de la investigación ya que sin su conocimiento tan amplio del tema del semillero «Optimización de la cadena de distribución del conglomerado Pymes del sector cárnico de Bogotá, 
D.C.» esto no hubiese sido posible de realizar y a Dios como guía permanente de nuestro proyecto, ya que ante las dificultades con todas las entidades para realizar nuestra encuestas él estuvo allí para forjarnos a terminarlas de la mejor manera.

\section{REFERENCIAS}

[1] Vatic Group, «Soluciones,» Vatic Group .

[2] J. Chavez, «Supply chain management.» Santiago: RiL editores, 2012.

[3] M. Birendra, R. Srinivasan and Y. Xiaohang, "Information sharing in supply chains: incentives for information distortion, v vol. 39, no. 9, pp. 863 877, 2007.

[4] Programa de Innovación Logística (Programa PILOT), «Las claves de la Suppy Chain.» Instituto Aragonés de fomento, 2002.

[5] R. Ballou, Logística administración de la cadena de suministro, Quinta edición. México: Pearson educación, 2004.

[6] S. Romero, S. Sepúlveda, «Territorio, agricultura y competitividad.» San José: IICA, 1999.

[7] A. Verdesoto, «Análisis de costos por actividades (Costeo $\mathrm{ABC}$ ) para el área de empaque secundario de un operador logístico en la ciudad de Quito,» Tesis de maestria, Universidad Andina Simón Bolívar, Ecuador, 2015.

[8] H.Mintzberg, J. Quinn, J. Voyer, El proceso estratégico: conceptos, contextos y casos, Primera. México: Pearson Prentice Hall, 1997.
[9] Universidad Dr. Jose Matias Delgado, «Posicionamiento de un Centro de Distribución como estrategia competitiva de las Empresas.» Ujmd, 2010.

[10] J. Antún, A. Lozano, J. Hernández, R. Hernández, Logística de Distribución Física a Minoristas, Serie Docencia. México: Instituto de Ingeniería UNAM.

[11] G. Svensson, «Gestión de la Cadena de Suministro frente a Gestión de la Cadena Sostenible.» EsicMarket.

[12] E. Frazelle, Suppy chain strategy. McGraw Hill Companies, 2002.

[13] European Logistics Association, «Empowering Logistics,» ELA.

[14] J. Anaya, «Logística Integral.» Madrid: ESIC, 2007.

[15] J. Thünen, von Thünen isolated state an english edition of Der Isolierte Staat, Translated By C. M. Wartenberg edited and introduced by P. Hall. 1966. Pergamon Press, 1826.

[16] Fortna, «Pasos para Alinear su Red de Distribución para Alcanzar Ventajas Competitivas.» 2012.

[17] Congreso de Colombia, Ley 590 de 2000. 2000, p. 16.

[18] Nutresa, «Informe ejecutivo.» 2013.

[19] A. Bustamante, «Eficacia de normativa de vertimientos en el sector cárnico.» Repositorio UDEM, 2012.

[20] FEDEGAN, «Consumo Aparente Per Cápita Anual,» Consumo Aparente, 2015. 